\title{
Fetal growth in low-risk Indian population at a tertiary centre and its comparison with INTERGROWTH-21 standards: a prospective cohort study
}

\author{
Anoosha K. Ravi ${ }^{1}$, Krishna Agarwal ${ }^{1 *}$, Siddarth Ramji ${ }^{2}$, Sreenivas Vishnubhatla ${ }^{3}$
}

\author{
${ }^{1}$ Department of Obstetrics and Gynecology, Maulana Azad Medical College, New Delhi, India \\ ${ }^{2}$ Department of Neonatology, Maulana Azad Medical College, New Delhi, India \\ ${ }^{3}$ Department of Biostatistics, All India Institute of Medical Sciences, New Delhi, India
}

Received: 09 September 2019

Accepted: 04 October 2019

\author{
*Correspondence: \\ Dr. Krishna Agarwal, \\ E-mail: drkrishna.agarwal@gmail.com
}

Copyright: (c) the author(s), publisher and licensee Medip Academy. This is an open-access article distributed under the terms of the Creative Commons Attribution Non-Commercial License, which permits unrestricted non-commercial use, distribution, and reproduction in any medium, provided the original work is properly cited.

\begin{abstract}
Background: The objective of this study was to compare the fetal growth pattern in low risk Indian population with the INTERGROWTH-21 standards.

Methods: Low risk women were enrolled at 10 to 20 weeks of gestation and followed up until delivery. An experienced operator performed abdominal ultrasound every $5 \pm 1$ week and measured biparietal diameter (BPD), head circumference (HC), abdominal circumference (AC) and femur length (FL) of the fetus. Newborn anthropometric measurements were taken within 12 hours of childbirth.

Results: A total of 126 healthy women, enrolled at mean gestation of $16.8 \pm 1.6$ weeks, completed the follow up until delivery. None of the participants developed any major obstetric or medical morbidity. The study subjects showed lower mean $\mathrm{z}$ scores for BPD $(-0.7 \pm 1.3)$, HC $(-0.4 \pm 1.3)$ and AC $(-0.4 \pm 1.3)$ but a higher mean $\mathrm{z}$-score for FL $(0.3 \pm 1.7)$ as compared to INTERGROWTH-21 standards. From $1^{\text {st }}$ through $5^{\text {th }}$ visit, the $\mathrm{z}$ scores for BPD and HC improved whereas declined for AC and FL.

Conclusions: The fetal growth in non-affluent healthy Indian women had a lower fetal growth compared to INTERGROWTH-21 standards.
\end{abstract}

Keywords: Abdominal circumference, Biparietal diameter, Femur length, Intrauterine fetal growth, Percentile charts, Ultrasound parameters

\section{INTRODUCTION}

The sources for percentile charts to guide intrauterine growth are normograms constructed from population in USA or Europe (e.g.; Alexander et al, Brenner et al,) or from mothers with known constraints to growth., ${ }^{1,2}$ Similar to the WHO reference standards available for growth monitoring from 0 to 5 years of age, a global initiative was taken to develop global reference standards for intrauterine fetal growth monitoring and was published as INTERGROWTH 21; International fetal and new-born growth consortium for the $21^{\text {st }}$ century. ${ }^{3,4}$ This was a prescriptive study involving affluent educated mothers. With this available international standard, the fetal growth of local population is studied which constitutes mostly socioeconomically disadvantaged mothers and thence the study will impress upon how fetuses from non-affluent population grow as compared to the international growth standards. 
Aim of this study was to study the fetal growth pattern and new-born size in low risk population attending a tertiary care teaching hospital and to compare it with the INTERGROWTH 21 standards.

\section{METHODS}

This was a prospective cohort study conducted at a tertiary care hospital, New Delhi, India. The study subjects were recruited from November 2014 till June 2015. The participants were followed and data collection was done till March 2016. The participants were pregnant women attending the antenatal clinic.

Women with known last date of menstrual cycle (LMP) with regular cycles and 10 to 20 weeks of pregnancy were included in the study. Regular period was defined as menstrual cycle of $28 \pm 4$ days. Gestational age (GA) was calculated from LMP and confirmed by measuring the crown rump length (CRL) between 10+0 weeks and 13+6 weeks and by bi-parietal diameter (BPD) between $13+6$ weeks and $20+0$ weeks. When GA by CRL or BPD was within 7 days of GA by LMP, LMP was used for dating. If GA by CRL or BPD was more than \pm 7 days of GA by LMP, those subjects were excluded from the study.

Women with multiple gestations, IVF pregnancy, major congenital anomaly (it was also planned to exclude the records of subjects if anomaly was detected after birth), medical disorders and women who were unlikely to remain in follow up were excluded from the study.

Pregnant women attending the antenatal clinic at gestational age between 10 and 20 weeks were screened for eligibility based on inclusion and exclusion criteria and were asked to participate in the study.

After inclusion into the study, subsequent scanning appointments were scheduled at approximately 5 weeks $( \pm 1$ week) interval. All the USG follow up visits were mostly coinciding with the routine follow up visits.

All the data were collected on a predesigned structured proforma. Ultrasound was performed using a transabdominal probe 3- 3.5 MHz (Philips HD 7).

At each visit, the measurements of BPD, HC, AC and FL were obtained 3 times from 3 separately generated ultrasound images. It was ensured that the images should fill at least $30 \%$ of the monitor screen. While doing ultrasound, the assessor was blinded to the previous readings to avoid bias.

BPD was measured from outer to outer edges of the parietal bones at the widest part of the skull in a crosssectional view of the fetal head at the level of the thalami. It was ensured that the image should be oval and symmetrical; the thalami were located symmetrically on each side of the midline falx.
HC was measured by placing the calipers on the outer border of the occipital and frontal edges of the skull at the point of the midline ('outer to outer') across the longest part of the skull. The HC was calculated from OFD and measurements using the ellipse facility.

AC was measured with the image of transverse section of the fetal abdomen as close as possible to circular with umbilical vein in its anterior third and stomach bubble visible in the image. It was ensured that kidneys and bladder were not be visible in the image.

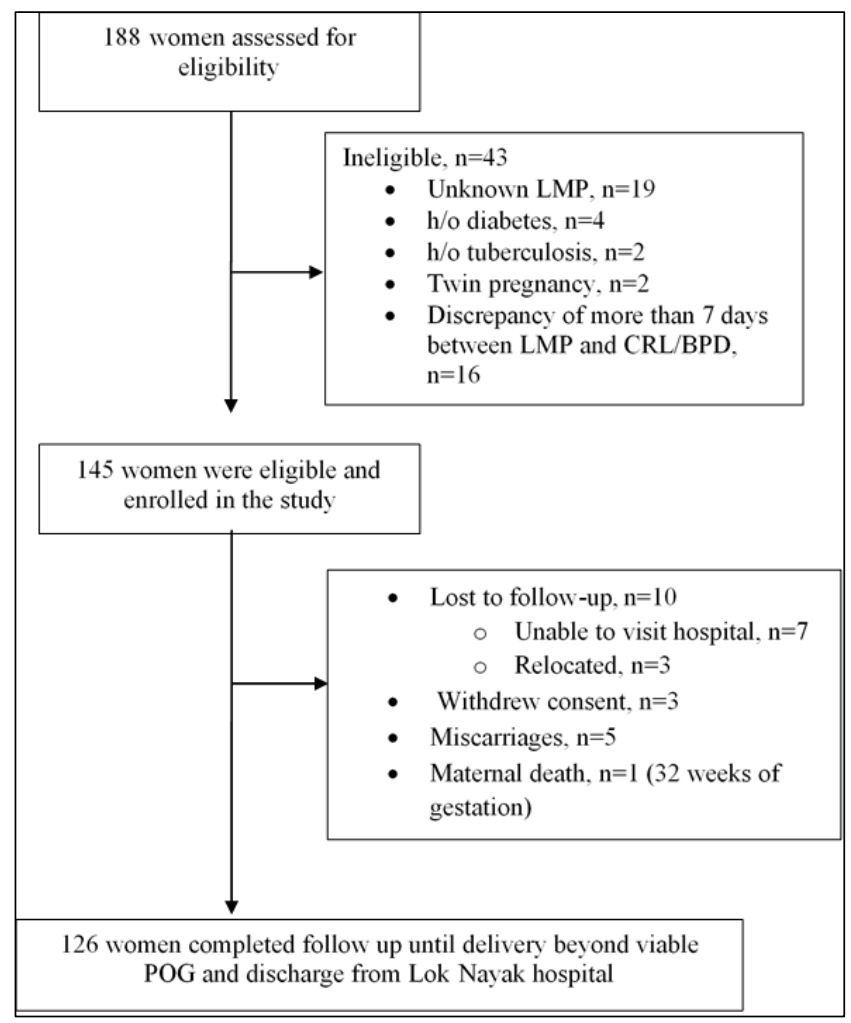

Figure 1: Study population flow chart.

FL was measured from outer to outer edges with the image as close as possible to the horizontal plane. The full length of the bone was visualized and trochanter was not measured.

Each scanning visit was accompanied by measurement of weight in $\mathrm{kg}$, blood pressure and fundal height. Iron, folate and calcium supplements were given. Adherence to the same was enquired in each visit. Adequacy of dietary intake was ensured by history by 24 hour recall method.

Parameters BPD, HC, AC and FL were checked for normality and expressed as Mean \pm SD. Percentile charts for $1^{\text {st }}, 10^{\text {th }}, 50^{\text {th }}, 90^{\text {th }}$ and $99^{\text {th }}$ percentiles were prepared. The resulting centiles were presented as trendlines.

\section{Statistical analysis}

Data was compared with INTERGROWTH 21 standards using two sample t-tests. $\mathrm{Z}$ score and $95 \%$ confidence 
interval of difference between study and intergrowth values were calculated using Stata, version 9.2 (Statacorp, Texas, USA). $\mathrm{P}$ value $<0.05$ was taken as significant.

Ethical clearance was taken from the institutional ethics committee.

\section{RESULTS}

A total of 188 women with pregnancy of 10 to 20 weeks gestation were assessed for eligibility (Figure 1). The study was conducted from November 2014 to April 2016. The interviewing and recruitment were done at the antenatal clinic and follow up visits were arranged coinciding the antenatal visit. The antenatal management was done as per the institution protocol. Finally, data from 126 women was analyzed.

Table 1 shows the baseline characteristics of the study population. These were healthy low risk women who belonged to lower socio-economic strata, modified Kuppuswamy class 3, 4 and 5.
Table 1: Baseline characteristics of enrolled women.

\begin{tabular}{|ll|}
\hline Characteristics & $\mathrm{N}=126$ \\
\hline Age; year & $24.6 \pm 3.4$ \\
\hline Height; cm & $155.1 \pm 5.9$ \\
\hline Body weight at first antenatal visit; kg & $52.9 \pm 10.1$ \\
\hline Weight less than $45 \mathrm{~kg}$ & $26(20.6)$ \\
\hline Body mass index (BMI), kg/m² & $21.8 \pm 3.3$ \\
\hline BMI<18 & $15(11.9)$ \\
\hline BMI $>25$ & $17(13.5)$ \\
\hline Formal education, completed years & $5.0 \pm 2.3$ \\
\hline Socio-economic status* & \\
\hline Class 3 & $74(58.7)$ \\
\hline Class 4 & $45(35.7)$ \\
\hline Class 5 & $7(5.5)$ \\
\hline Gestation at $1{ }^{\text {st }}$ antenatal visit, wk & $16.8 \pm 1.6$ \\
\hline Hemoglobin level at first visit, g/dL & $9.0 \pm 1.0$ \\
\hline Hb<6 g/dL & 0 \\
\hline Nulliparous & $92(73)$ \\
\hline Data expressed as Mean $\pm S D$ or number $(\%) ; ~$ & Modified \\
\hline Kuppuswamy scale. & \\
\hline
\end{tabular}

Table 2: Z (SDS) score for BPD, HC, AC and FL in comparison with INTERGROWTH-21.

\begin{tabular}{|c|c|c|c|c|c|}
\hline \multirow{2}{*}{ POG } & \multirow{2}{*}{$\mathbf{N}$} & \multicolumn{4}{|c|}{ Z score \pm SD } \\
\hline & & BPD & HC & $\mathbf{A C}$ & FL \\
\hline 14 & 14 & $-1.3 \pm 1.7$ & $-0.1 \pm 1.6$ & $-0.2 \pm 1.5$ & $0.8 \pm 1.9$ \\
\hline 15 & 11 & $-1.3 \pm 1.6$ & $-0.7 \pm 1.3$ & $-0.6 \pm 1.8$ & $0.6 \pm 1.9$ \\
\hline 16 & 30 & $-1.1 \pm 1.6$ & $-0.8 \pm 1.5$ & $-0.1 \pm 1.4$ & $0.2 \pm 2.0$ \\
\hline 17 & 21 & $-0.9 \pm 1.1$ & $-0.7 \pm 1.3$ & $-0.1 \pm 1.6$ & $0.2 \pm 1.7$ \\
\hline 18 & 20 & $0.1 \pm 1.1$ & $-0.1 \pm 1.3$ & $0.3 \pm 1.5$ & $0.7 \pm 1.8$ \\
\hline 19 & 30 & $-0.4 \pm 1.4$ & $-0.3 \pm 1.1$ & $-0.1 \pm 1.3$ & $0.7 \pm 1.3$ \\
\hline 20 & 21 & $-0.6 \pm 1.1$ & $-0.1 \pm 1.0$ & $0.1 \pm 1.0$ & $1.1 \pm 1.3$ \\
\hline 21 & 24 & $-1.3 \pm 1.6$ & $-1.1 \pm 1.3$ & $-0.7 \pm 1.2$ & $0.4 \pm 2.1$ \\
\hline 22 & 19 & $-0.5 \pm 1.7$ & $-0.5 \pm 1.5$ & $-0.2 \pm 1.4$ & $0.5 \pm 2.1$ \\
\hline 23 & 21 & $-1.1 \pm 1.4$ & $-1.1 \pm 1.5$ & $-0.7 \pm 1.1$ & $-0.4 \pm 2.0$ \\
\hline 24 & 19 & $0.1 \pm 1.4$ & $0.3 \pm 0.9$ & $0.1 \pm 1.2$ & $1.3 \pm 2.0$ \\
\hline 25 & 22 & $-0.9 \pm 1.2$ & $-0.6 \pm 1.3$ & $-0.3 \pm 1.3$ & $0.6 \pm 1.3$ \\
\hline 26 & 25 & $-0.5 \pm 1.3$ & $-0.2 \pm 1.2$ & $-0.2 \pm 1.2$ & $0.3 \pm 1.5$ \\
\hline 27 & 29 & $-1.0 \pm 1.4$ & $-0.8 \pm 1.2$ & $-0.7 \pm 1.4$ & $-0.3 \pm 1.6$ \\
\hline 28 & 19 & $-0.4 \pm 1.3$ & $-0.6 \pm 1.2$ & $-0.4 \pm 1.1$ & $0.1 \pm 2.2$ \\
\hline 29 & 21 & $-0.2 \pm 1.1$ & $-0.1 \pm 1.1$ & $0.1 \pm 1.3$ & $0.6 \pm 1.4$ \\
\hline 30 & 22 & $-0.5 \pm 1.1$ & $-0.1 \pm 1.1$ & $-0.4 \pm 1.3$ & $0.6 \pm 1.5$ \\
\hline 31 & 22 & $-0.5 \pm 1.2$ & $-0.4 \pm 1.3$ & $-0.5 \pm 1.3$ & $0.3 \pm 1.2$ \\
\hline 32 & 30 & $-0.8 \pm 1.2$ & $-0.4 \pm 1.0$ & $-0.5 \pm 1.2$ & $0.1 \pm 1.2$ \\
\hline 33 & 27 & $-0.7 \pm 1.0$ & $-0.5 \pm 1.1$ & $-0.6 \pm 1.1$ & $-0.2 \pm 1.5$ \\
\hline 34 & 16 & $-0.5 \pm 1.2$ & $-0.2 \pm 1.0$ & $-0.3 \pm 1.4$ & $0.3 \pm 1.4$ \\
\hline 35 & 19 & $-0.5 \pm 1.3$ & $-0.1 \pm 1.4$ & $-0.3 \pm 1.3$ & $0.3 \pm 1.6$ \\
\hline 36 & 24 & $-0.4 \pm 1.0$ & $-0.2 \pm 1.2$ & $-0.4 \pm 1.2$ & $0.4 \pm 1.2$ \\
\hline 37 & 10 & $-0.6 \pm 1.6$ & $-0.5 \pm 1.4$ & $-1.3 \pm 1.1$ & $0.1 \pm 1.0$ \\
\hline 38 & 3 & $-1.2 \pm 0.4$ & $-0.7 \pm 1.0$ & $-1.7 \pm 1.0$ & $-0.2 \pm 0.5$ \\
\hline \multirow[t]{2}{*}{39} & 4 & $-0.3 \pm 1.2$ & $-0.1 \pm 0.5$ & $-0.4 \pm 1.5$ & $-0.2 \pm 1.6$ \\
\hline & & $\mathrm{BPD}$ & $\mathrm{HC}$ & $\mathrm{AC}$ & $\mathrm{FL}$ \\
\hline \multicolumn{2}{|c|}{ MEAN Z score } & $-0.7 \pm 1.3$ & $-0.4 \pm 1.3$ & $-0.4 \pm 1.3$ & $0.3 \pm 1.7$ \\
\hline
\end{tabular}

Data expressed as Mean \pm SD., BPD, bipariental diameter; HC, head circumference; AC, abdominal circumference; FL, femur length; SDS, standard deviation score. 
On an average, each subject had 4 visits at $5 \pm 1$ weeks interval. The visits had been grouped into 5 sets as follows- first visit at $10+0$ to $19+6$ weeks of gestation, second visit at $20+0$ to $24+6$ weeks of gestation, third visit at $25+0$ to $29+6$ weeks of gestation, fourth visit at 30 to $34+6$ weeks of gestation and the fifth visit at 35 to 40 weeks of gestation.

Mean and SD for all four parameters, the BPD, HC, AC and the FL, were measured at every visit. At 20 weeks POG, the mean and SD for BPD, HC, AC and FL were $48.4 \pm 3.0 \mathrm{~mm}, \quad 177.6 \pm 8.8 \mathrm{~mm}, 148.1 \pm 7.4 \mathrm{~mm}$ and $33.3 \pm 2.4 \mathrm{~mm}$ respectively. At 30 weeks POG the mean and SD for same parameters were $78.3 \pm 3.6 \mathrm{~mm}$, $281.0 \pm 11.0 \mathrm{~mm}, 248.8 \pm 16.7 \mathrm{~mm}$ and $56.8 \pm 3.3 \mathrm{~mm}$ respectively. There was steady growth in BPD, HC, FL and $\mathrm{AC}$ as the gestation advanced. However, the rate of growth slowed down as the gestation approached near term. Beyond 37 weeks, the measurements do not follow the pattern and in fact at 38 weeks the measurements are smaller than the ones at 37 weeks. This may be due two reasons. One, due to a smaller number of observations (3 at 38 weeks and 4 at 39 weeks). Second, it is difficult to take measurements correctly due to overcrowding of fetal parts.

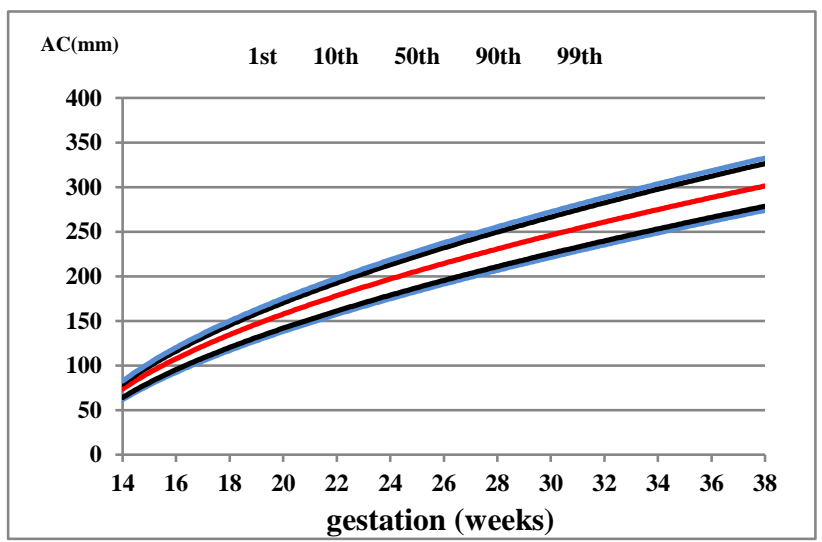

Figure 2: Percentiles of fetal abdominal circumference in study population.

Table 3: Z score (SDS) for BPD, HC, AC and FL in comparison with INTERGROWTH-21.

\begin{tabular}{|c|c|c|c|c|c|c|}
\hline \multirow{2}{*}{ Visit } & \multirow{2}{*}{$\mathbf{N}$} & \multirow{2}{*}{ Mean gestation (weeks) } & \multicolumn{4}{|l|}{$\mathbf{Z}$ score } \\
\hline & & & BPD & HC & $\mathbf{A C}$ & FL \\
\hline $1^{\text {st }}$ & 126 & 16.9 & $-0.8 \pm 1.5$ & $-0.5 \pm 1.4$ & $-0.1 \pm 1.5$ & $0.5 \pm 1.7$ \\
\hline $2^{\text {nd }}$ & 104 & 21.9 & $-0.7 \pm 1.5$ & $-0.5 \pm 1.4$ & $-0.3 \pm 1.2$ & $0.4 \pm 2.0$ \\
\hline $3^{\text {rd }}$ & 116 & 26.9 & $-0.6 \pm 1.3$ & $-0.5 \pm 1.2$ & $-0.4 \pm 1.3$ & $0.2 \pm 1.6$ \\
\hline $4^{\text {th }}$ & 117 & 31.9 & $-0.6 \pm 1.2$ & $-0.3 \pm 1.1$ & $-0.5 \pm 1.2$ & $0.2 \pm 1.4$ \\
\hline $5^{\text {th }}$ & 60 & 36.2 & $-0.5 \pm 1.2$ & $-0.2 \pm 1.3$ & $-0.6 \pm 1.3$ & $0.2 \pm 1.3$ \\
\hline
\end{tabular}

Data expressed as Mean \pm SD; BPD, biparietal diameter; HC, head circumference; AC, abdominal circumference; FL, femur length;

SDS, standard deviation score.

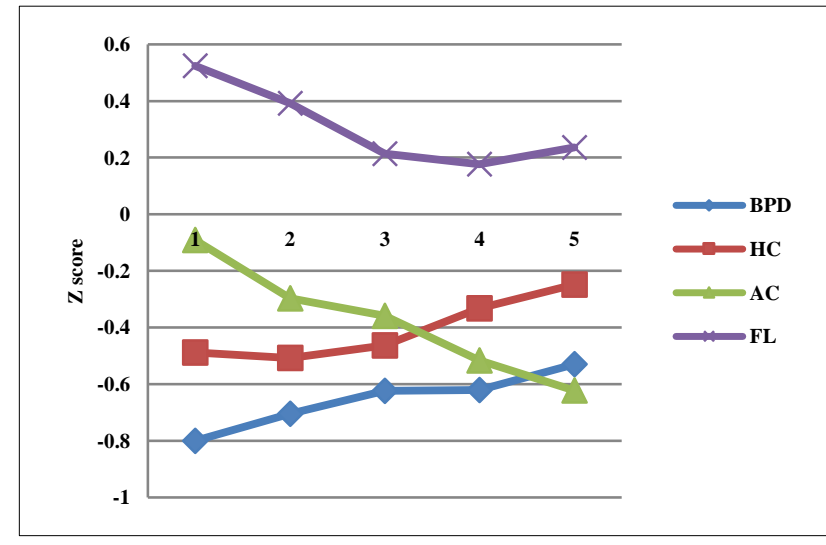

Figure 3: Mean z score in comparison with intergrowth-21.

Percentiles of AC $\left(1^{\text {st }}, 10^{\text {th }}, 50^{\text {th }}, 90^{\text {th }}\right.$ and $\left.99^{\text {th }}\right)$ are shown in the graphical form in Figure 2. These figures suggest that data is normally distributed. The data is presented as power trendlines generated in excel sheet with $\mathrm{R}$ squared value (coefficient of determination) as 0.986 which is an almost perfect fit of the line to the data.

\section{Comparison with INTERGROWTH 21 study}

Our study population showed lower mean $\mathrm{z}$ scores for BPD, HC and AC as per INTERGROWTH-21standards. However mean $\mathrm{z}$ score of FL was higher when compared to INTERGROWTH-21standards (Table 2). To understand the trend of $\mathrm{Z}$ score, the sets of visits were analysed. $\mathrm{Z}$ scores of BPD and HC improved from $1^{\text {st }}$ through fifth visit (Table 3, Figure 3) but the same for AC declined further from $1^{\text {st }}$ to $5^{\text {th }}$ visit. In case of FL, a decline was noted from $1^{\text {st }}$ through fifth visit.

\section{DISCUSSION}

This study demonstrates that fetal growth in the study population is lower as compared to the INTERGROWTH-21standards. The mean z-score of three of four fetal parameters namely BPD, HC and AC had a value of around -0.5 at all 5 time points starting 
from 14 weeks of gestation until delivery. A total of 39 subjects had $\mathrm{AC}$ value below $10^{\text {th }}$ percentile.

This study was planned in hospital setting and the study population belonged to lower socio-economic strata. However, we attempted to exclude women with other factors known to constrain fetal growth such as occurrence of gestational hypertension. Consequently, the rates of such morbidities were low in our study cohort. The BMI at first visit was $21.8 \mathrm{~kg} / \mathrm{m}^{2}$ however, like Indian participants in INTERGROWTH-21 study cohort, height of women in our study cohort was low. ${ }^{5}$ It can be argued that the difference in the two study populations with respect to some characteristics such as SES and maternal height, may have been responsible for lower fetal growth in our study.

INTERGROWTH-21 study provided fetal growth standards that is recommended across the globe. The INTERGROWTH-21 study investigators argued that the fetal growth is uniform across the globe irrespective of ethnicity once the socio-economic, environmental and health related constraints to fetal growth are removed. INTERGROWTH-21 standards are increasingly being adopted by more and more countries.

The closer look of INTERGROWTH-21 data, however, does show a relatively lower fetal growth in Indian women. The fetal growth parameters, CRL and HC had a mean $\mathrm{z}$ score which was around -0.5 and actually $\mathrm{HC}$ had $\mathrm{z}$ score of -0.58 between 34 weeks and 40 weeks of gestation. The Indian women had different baseline characteristics compared to the overall cohort. The mean Height of the Indian cohort was $158.3+3.6$ while it was $162.2+3.8 \mathrm{~mm}$ in overall cohort. The mean birthweight of newborn born at 37 weeks or more gestation for Indian cohort was $2.9 \mathrm{~kg}$ compared to $3.3 \mathrm{~kg}$ for the entire cohort. The authors however disregarded these differences and argued for the concept of uniform fetal growth in a bigger picture. Our study shows that fetal growth parameters (BPD, HC and AC) were lower than the pooled cohort of INTERGROWTH-21 study but actually were quite similar to that of Indian women in INTERGROWTH-21 study.

Why Indian women in INTERGROWTH-21 study had a lower fetal growth compared to the women from rest of the world in INTERGROWTH-21 study? Did they have factors that constrained their fetal growth? Indeed, that may be the case. As it was referred earlier, the height of Indian women in INTERGROWTH study was lower than overall cohort. Height could be a factor that reflects malnutrition of the mother accrued over generations and therefore the same got transmitted to their fetuses also. It was reported that the Indians migrating to the USA had lower birthweight despite belonging to high socioeconomic class and having good nutrition our study cohort also had a lower height and had additional constraint in terms of lower SES. ${ }^{6}$ However, fetal growth and birthweight was quite comparable to Indian cohort of
INTERGROWTH-21study. It highlights the complexity of the issues involved in fetal growth and their inadequate understanding.

The socio-economic status may be proxy for a variety of factors such as income level, education, occupation, housing, sanitation, intercurrent illnesses; to name a few. Though there are many studies supporting the constraint in fetal growth in the population with low socioeconomic status, none can pinpoint on a particular factor and how it affects fetal growth remains to be revealed. ${ }^{6}$

In our cohort, FL consistently has higher mean z-score value $(+0.3$ at all-time points). The linear growth parameters in INTERGROWTH-21 study did not show this finding in Indian women. We employed standardized methods for the measurement of this parameter and therefore measurement bias is unlikely. It may be a chance finding due to small sample size and may not have much significance.

Has time come to shift from customized charts based on the maternal height, weight and the ethnic origin to use international growth standards, still needs to be further validated. $^{7,8}$

Limitation of our study is small sample size. The number of visits were small (every $5 \pm 1$ week) therefore events between 5 weeks window could have been missed.

\section{CONCLUSION}

Strengths of our study included its prospective cohort design, robust methodology, no interobserver bias. The ultrasound operator was blinded to gestation of the women preventing any bias in this regard.

\section{ACKNOWLEDGMENTS}

Authors would like to thank Dr. Reva Tripathi for her valuable suggestions.

Funding: No funding sources

Conflict of interest: None declared

Ethical approval: The study was approved by the Institutional Ethics Committee

\section{REFERENCES}

1. Alexander GR, Himes JH, Kaufman RB, Mor J, Koqan M. A united states national reference for fetal growth. Obstet Gynaecol. 1996;87(2):163-8.

2. Brenner WE, Edelman DA, Hendricks CG. A standard of fetal growth for the United States of America. Am J Obstet Gynaecol. 1976;126:555.

3. De Onis M, Martorell R, Garza C, Lartey A. WHO child growth standards based on length/height, weight and age. Acta Paediatrica. 2006;450:76-85.

4. Papageorghiou AT, Ohuma EO, Altman DG, Todros $\mathrm{T}$, Ismail LC, Lambert $\mathrm{A}$, et al. International 
standards for fetal growth based on serial ultrasound measurements: the fetal growth longitudinal study of the INTERGROWTH-21st project. The Lancet. 2014;384(9946):869-79.

5. Villar J, Papageorghiou AT, Pang R, Ohuma EO, Ismail LC, Barros FC, et al. The likeness of fetal growth and newborn size across non-isolated populations in the INTERGROWTH-21st Project: the fetal growth longitudinal study and newborn cross-sectional study. The Lancet Diab Endocrinol. 2014;2(10):781-92.

6. Gould JB, Madan A, Qin C, Chavez G. Perinatal outcomes in two dissimilar immigrant populations in the United States: a dual epidemiologic paradox. Pediatr. 2003;111(6 Pt 1):e676-82.

7. Gardosi J, Figueras F, Clausson B, Francis A. The customised growth potential:an international research tool to study the epidemiology of fetal growth. Paediatr Perinat Epidemiol. 2011;25(1):2-10.

8. Unterscheider J, Geary MP, Daly S, McAuliffe FM, Kennelly MM, Dornan J, et al. The customized fetal growth potential: a standard for Ireland. Eu J Obstet Gynecol Repro Biol. 2013 Jan 1;166(1):14-7.

Cite this article as: Ravi AK, Agarwal K, Ramji S, Vishnubhatla S. Fetal growth in low-risk Indian population at a tertiary centre and its comparison with INTERGROWTH-21 standards: a prospective cohort study. Int J Reprod Contracept Obstet Gynecol 2019;8:4400-5. 